\title{
On the temporal variability classes found in long gamma-ray bursts with known redshift
}

\author{
L. Borgonovo ${ }^{1}$, F. Frontera ${ }^{2}$, C. Guidorzi ${ }^{2,3,4,5}$, E. Montanari ${ }^{2}$, L. Vetere ${ }^{6,7}$, and P. Soffitta ${ }^{7}$
}

\author{
${ }^{1}$ Stockholm Observatory, 10691 Stockholm, Sweden \\ e-mail: luis@astro.su.se \\ 2 Dipartimento di Fisica, Università di Ferrara, 44100 Ferrara, Italy \\ 3 Astrophysics Research Institute, Liverpool John Moores University, Twelve Quays House, Birkenhead CH41 1LD, UK \\ 4 Dipartimento di Fisica, Università di Milano-Bicocca, Italy \\ 5 INAF, Osservatorio Astronomico di Brera, via Bianchi 46, 23807 Merate (LC), Italy \\ 6 Dipartamento di Fisica, Università La Sapienza, Piazzale A. Moro 2, 00185 Roma, Italy \\ 7 INAF, IASF - Sezione di Roma, via del Fosso del Cavaliere, 00133 Roma, Italy
}

Received 20 October 2006 / Accepted 9 January 2007

\section{ABSTRACT}

\begin{abstract}
Context. Based on the analysis of a small sample of BATSE and Konus gamma-ray bursts (GRBs) with know redshift it has been reported that the width of the autocorrelation function (ACF) shows a remarkable bimodal distribution in the rest-frame of the source. However, the origin of these two well-separated ACF classes remains unexplained.

Aims. We study the properties of the bursts belonging to each ACF class and look for significant differences between them.

Methods. We complement previous ACF analysis studying the corresponding power density spectra (PDS). With the addition of Beppo-SAX data and taken advantage of its broad-band capability, we not only increase the burst sample but we extend the analysis to X-ray energies.

Results. The rest-frame PDS analysis at $\gamma$-ray energies shows that the two ACF classes are not simply characterised by a different low frequency cut-off, but they have a distinct variability as a whole in the studied frequency range. Both classes exhibit average PDS with power-law behaviour at high frequencies $\left(f^{\prime} \geq 0.1 \mathrm{~Hz}\right)$ but significantly different slopes, with index values close to those of Brownian (-2) and Kolmogorov (-5/3) spectra for the narrow and broad classes respectively. The latter spectrum presents an additional PDS component, a low-frequency noise excess with a sharp cut-off at a characteristic frequency $f_{c}^{\prime} \simeq 0.025 \mathrm{~Hz}$, in conjunction with the small relative dispersion $(\sim 6 \%)$ of the ACF width in this class. At X-ray energies we find the power-law index unchanged for the broad class, but a significantly steeper slope in the narrow case ( $\sim-3)$. We interpret this as an indication that the broad class bursts have weaker spectral evolution than the narrow ones, as suggested also by our analysis of the ACF energy dependence. The low and high frequency PDS components may then arise from two radiating regions involving different emission mechanisms. We compare our GRB sample conditioned by afterglow detections with a complete, flux limited BATSE sample, finding a significant bias against narrow ACF bursts.
\end{abstract}

Key words. gamma rays: bursts - gamma rays: observations - methods: data analysis - cosmology: distance scale

\section{Introduction}

Determining the relevant timescales for any astronomical phenomenon is essential to understand its underlying physical processes. However, in spite of extensive research, temporal studies on the prompt emission phase of long gamma-ray bursts (GRBs) are not yet able to describe and explain their basic temporal properties. The main challenges encountered in the temporal analysis of GRBs are related to intrinsic characteristics of the emitted signal. Firstly, bursts are non-repetitive short-term events. Consequently, the total duration of the emission in a given observational energy window is the first timescale used to characterised them. Through out this paper we will only consider the class of long bursts, i.e., those with duration time $T_{90}>2 \mathrm{~s}$. Second, burst light curves (LCs) show a remarkable morphological diversity and they appear to have a composite structure. While a significant fraction of bright long bursts $(\sim 15 \%)$ exhibits a single smooth pulse structure, in most cases they appear to be the result of a complex, seemingly random distribution of several pulses. Burst pulses are commonly described as having fast-rise exponential-decay (FRED) shape, although the decay is not strictly exponential. Therefore, the second timescale that seems relevant for the description of a burst is a "typical" pulse duration. However, analysis of the pulse parameters has shown broad log-normal distributions not only among different bursts, but also within a single burst (see, e.g., Norris et al. 1996). Consideration of other timescales might be relevant, e.g., for GRBs with precursors (Koshut et al. 1995) or when long quiescent periods occur (Nakar \& Piran 2002), although these temporal features appear only in a small fraction of bursts.

Due to these characteristics, much of the GRB temporal analysis has been done directly on the LCs, i.e., modelling the pulses and studying their shape and distribution. Standard linear analysis tools must be used with some caution, since most of the inferences based on them would in principle require "long" stationary signals, i.e., the most suitable bursts are those where the duration is much longer than the typical pulse width. This is the case of the temporal analysis based on power density spectra (PDS). Individual PDS of GRB have very diverse shapes, and they do not seem to have common features, although the longest 
bursts show spectra with a consistent power-law behaviour. One way to overcome these limitations is to estimate an average PDS from a sample GRBs. This approach will only produce physically meaningful results if each burst can be considered a realisation of the same stochastic process, i.e., there are no subclasses in the sample.

Under this assumption Beloborodov et al. (1998, 2000) calculated with a large sample of bright GRBs, an average PDS showing a clear power-law behaviour extended over two frequency decades (approximately within the $0.01-1 \mathrm{~Hz}$ frequency range), and more remarkably with an exponent value approximately equal to that of the Kolmogorov spectrum found in fluid turbulence (Kolmogorov 1941). The significance of a thus obtained average PDS depends on two important additional factors. First, light curves have to be normalised to balance the weight between bursts of different brightness. It is not clear at this point which norm should be used to produce the most meaningful average. Beloborodov et al. (1998) favoured the use of the peak flux normalisation, however they tested several other norms with qualitatively similar results. Thus, a good normalisation should give better convergence but the norm should not affect the final result for a sufficiently large sample. The second problem is the shift in frequencies produced by cosmic dilation effects. Having no redshifts $z$ determined for their GRB sample, Beloborodov et al. (2000) did not correct for these effects. However, they argued that if the underlying PDS shape for every GRB is a featureless single power-law with a constant exponent, the frequency shifts will not affect the obtained average PDS. If this is the case, considering that the two decades range of the power-law is much larger than the standard deviation of the redshift distribution ( $\sigma_{z} \sim 2$ based on the few known redshifts), indeed the shifts should just smear the cut-off frequencies.

The same statistical approach was used by Fenimore et al. (1995, hereafter F95) in their study of the average autocorrelation function (ACF) of a sample of bright GRBs. The ACF gives a measure of the correlation between different points in the light curve that are separated by a given time lag. Since it is the Fourier transform of the PDS, it contains in principle the same information that can be visualised in a different way. Therefore, the same caveats regarding the average PDS apply to the average ACF. It was only after the discovery of the afterglow emission (Costa et al. 1997) and the determination of their redshift for a significant number of bursts that we were able to address some of those issues. Borgonovo (2004, hereafter B04) showed for a sample of 16 bright GRB with known $z$ that when corrected for cosmic dilation effects the ACFs exhibit a clear bimodal distribution. Using as a measure the half-width at half-maximum, there is a highly significant gap between a narrow and a broad width class, the separation in standard deviations being $>7 \sigma$. The estimated local or intrinsic values (i.e., those calculated at the restframe of the source) for the average widths were $1.6 \mathrm{~s}$ and $7.5 \mathrm{~s}$, and the relative dispersions were $32 \%$ and $4 \%$ for the narrow and broad classes, respectively. It is remarkable the low dispersion found in the last subset, which comprised $\sim 1 / 3$ of the total sample.

This article builds on the ACF analysis done in B04. In Sect. 2 we present our data samples and in Sect. 3 we briefly describe the methods used in the subsequent temporal analysis. In Sect. 4 we strengthen previous findings based on the ACF, expanding the previous sample of GRB with known $z$ by the inclusion of proprietary data from the BeppoSAX mission, and we complement the temporal analysis estimating the intrinsic PDS for each of the subsets identified using the ACF. Furthermore, using the broadband capability of BeppoSAX combined instruments, in Sect. 5 we are able to extend the study to the Xray energies. In Sect. 6, we investigate whether the typical values of several physical parameters commonly used to characterised GRBs differ significantly between the two temporal classes. We look into the problem of the energy dependence of the ACF and discuss possible biases in our sample of bursts with known $z$ in Sect. 7. In Sect. 8 we discuss our main results.

\section{Data}

This work is mainly based on the analysis of light curves from GRBs with known redshift. Given the scarce number of cases available for study, we combined data (in the gamma energy band) from three instruments to improve our statistics. Increasing a sample in this way presents an obvious trade-off, since we use count time series and the difference between instrument responses introduce an additional dispersion that may counteract the benefits. For this reason, we initially analysed the data of the bursts that were observed by more than one instrument, evaluating whether the differences were acceptable for our purposes.

The comparisons were made taking BATSE as the reference instrument. Its data comprise half of our GRB sample (in the gamma energy band), showing the best signal-to-noise ratio $(\mathrm{S} / \mathrm{N})$ with relatively low directional dependence thanks to the large collecting area of its eight Large Area Detectors (LADs) placed on each corner of the Compton Gamma-Ray Observatory (CGRO, Fishman et al. 1989), giving full sky coverage. It flew during the period 1991-2000 collecting the largest GRB cata$\log$ up to date. The CGRO Science Support Center (GROSSC) provides the so-called concatenated $64 \mathrm{~ms}$ burst data, which is a concatenation of the three standard BATSE data types DISCLA, PREB, and DISCSC. All three data types have four energy channels (approximately 25-55, 55-110, 110-320, and >320 keV). The DISCLA data is a continuous stream of $1.024 \mathrm{~s}$ and the PREB data covers the $2.048 \mathrm{~s}$ prior to the trigger time at $64 \mathrm{~ms}$ resolution, both types obtained from the 8 LADs. They have been scaled to overlap the DISCSC $64 \mathrm{~ms}$ burst data, that was gathered by the triggered LADs (usually the four closer to the line of sight). This combined data format was used when available, since the concatenated pre-burst data allows a better estimation of the background. In the case of GRB 970828 the DISCSC data are incomplete, and we used instead the 16-channel MER data type, binned up into 4 DISCSC-like energy channels. All BATSE bursts with known $z$ were considered for study, excluding two cases were the data are incomplete or were no recorded (i.e., GRB 980326 and GRB 980613), resulting in a total of 11 cases.

We also include the set of bursts selected in B04 that were observed by Konus, which is a GRB detector on board the Wind mission (Aptekar et al. 1995). Light curves of its bursts are publicly available at $64 \mathrm{~ms}$ resolution in the 50-200 keV energy band. The collecting area of this experiment is about 20 times smaller than the one of BATSE and consequently, in most cases, the signal is too weak for our temporal studies. However, in B04 a comparative ACF analysis of the bursts observed by both instruments showed a good agreement for very bright bursts, and the selection criteria for Konus cases were set requiring peak count rates larger than 3000 counts $\mathrm{s}^{-1}$ and the availability of post-burst data, resulting in the 5 Konus GRBs included in B04 burst sample.

The BeppoSAX mission, that operated between the years 1996-2002, had broad energy band capabilities thanks to the combined operation of several instruments. The Gamma Ray 
Burst Monitor (GRBM; see Frontera et al. 1997) covered the 40-700 keV energy range, roughly matching the range of Konus and BATSE (i.e., 55-320 keV using $2+3$ channels). Note that since the ACF and the PDS are quadratic functions of the number of counts, and generally there are more counts at lower energies, the agreement for these temporal analysis functions will depend mainly on having a similar lower-end energy limit. We made use of the standard high resolution $7.8125 \mathrm{~ms}$ LCs, but we binned them up into a $62.5 \mathrm{~ms}$ time resolution to improve the $\mathrm{S} / \mathrm{N}$ ratio. This has a negligible effect on the measurement of the time scales that concern us here and it reduces some noise artifacts (i.e., the noise becomes more Poissonian). In addition, it approximately matches the standard $64 \mathrm{~ms}$ BATSE temporal resolution for better comparison. The LCs were dead-time corrected and background subtracted. In Borgonovo et al. (2005) a comparative ACF analysis was presented, including 17 GRBS detected by the GRBM with known redshift from which 8 were also observed by BATSE. It was concluded there that although the measured dispersions of the local ACF widths were larger than in the BATSE case ( $\sim 15 \%$ at half-maximum), the average sample values for each ACF width class were equal to within uncertainties. Therefore, at least in the context of the present temporal variability analysis, we can consider that these data have more intrinsic dispersion, but the introduced errors are mainly stochastic.

The two Wide Field Cameras (WFCs) also on board BeppoSAX covered the $2-26 \mathrm{keV}$ energy range (Jager et al. 1997). During their operation time they detected 53 GRBs in conjunction with the GRBM allowing the first broad band studies of GRBs (see, e.g., Amati et al. 2002). Furthermore, a considerable fraction of the WFC bursts $(\sim 36 \%)$ were also detected by BATSE. The LCs were extracted with a time resolution of $62.5 \mathrm{~ms}$ and discriminated in three energy channels (i.e., 2-5, 5-10, and 10-26 keV). The energy intervals were chosen in order to have a similar amount of counts in each channel for a typical GRB. However, except for the brightest GRBs, the signal in each channel is too weak for the purposes of our temporal analysis and the LCs had to be integrated into a single energy channel. Here we will focus on the analysis of the 13 GRBs for which we have redshift estimations. These bursts constitute a subset of our sample of GRBs with known redshift in the $\gamma$ energy band. Table 1 lists all these bursts indicating in its first columns their name, the source instruments in $\gamma$ and X-ray bands when available, the estimated redshift $z$, and the corresponding reference.

To further study the ACF width energy dependence we selected from the BATSE current catalog all long bursts (i.e., duration time $T_{90}>2 \mathrm{~s}$ ) with a peak flux measured on the $1.024 \mathrm{~s}$ timescale $F_{1 \mathrm{~s}} \geq 4$ photons $\mathrm{cm}^{-2} \mathrm{~s}^{-1}$ in the $50-300 \mathrm{keV}$ band that have available concatenated $64 \mathrm{~ms}$ data. This resulted in a sample of 188 bright bursts for which, in most cases, the redshift is unknown.

\section{Methods}

For the autocorrelation function analysis we follow the same method presented in B04, that was based on earlier works of Link et al. (1993) and F95. Here, we will summarise the method, and we refer to B04 for further details. Following the same notation, from a uniformly sampled count history with $\Delta T$ time resolution and $N$ time bins, let $m_{i}$ be the total observed counts at bin $i$. Also let $b_{i}$ be the corresponding background level and $c_{i}=m_{i}-b_{i}$ the net counts. The discrete ACF as a function of the time lag $\tau=k \Delta T$ is

$A(\tau=k \Delta T) \equiv \sum_{i=0}^{N-1} \frac{c_{i} c_{i+k}-m_{i} \delta_{0 k}}{A_{0}}, \quad k=0, \ldots, N-1$,

where $\delta$ is the Kronecker function. Here the periodic boundary conditions $\left(c_{i}=c_{i+N}\right)$ are assumed. The normalisation constant $A_{0}$ is defined as

$A_{0} \equiv \sum_{i=0}^{N-1}\left(c_{i}^{2}-m_{i}\right)$

such that $A(0)=1$ for $k=0$. The term $m_{i}$ in Eq. (2) subtracts the contribution of the uncorrelated noise assuming that it follows the Poisson statistics.

For practical reasons, the actual calculation of Eq. (1) was done using a Fast Fourier Transform (FFT) routine. Denoting by $C_{f}$ the Fourier transformed of the background subtracted light curve, then the definition of the power density spectrum (PDS) can be written as $P_{f} \equiv\left|C_{f}\right|^{2}$. The noise contribution is subtracted from the PDS assuming Poisson statistics. Since the PDS and the ACF are Fourier pairs (Wiener-Khinchin theorem), the latter is obtained by inverse transforming the first. Zero padding of the time series was used to avoid the artifacts produced by the periodic boundary condition. The normalisation used for the $\mathrm{ACF}$, that gives to its central maximum unity value, is equivalent (aside from a noise correction term) to the scaling of the LC by the square root of total power $\sqrt{P_{\text {tot }}}$, where $P_{\text {tot }} \equiv \sum_{i} c_{i}^{2}$. This normalisation is a natural choice for the ACF analysis and it makes the ACF of each burst independent (to first order) of its brightness. Note however that for our PDS analysis we found it more suitable to scale the LCs by their respective net count fluences $\mathcal{F} \equiv \sum_{i} c_{i}$ (or equivalently dividing the PDS of the original LCs by $\mathcal{F}^{2}$ ). Since the $a_{0}$ zero order Fourier coefficient is equal to the fluence $\mathcal{F}$, our normalisation makes the PDS converge to unity towards the low frequencies, therefore in Fourier space it can be interpreted also as normalising by the amount of power at the lowest frequency. In Sect. 4.2 we will further discuss this choice over other possible normalisations.

The background estimations were done by fitting with up to a second order polynomial the pre- and post-burst data, that were judged by visual inspection to be inactive. This is critical for weak bursts and particularly aggravated in the Konus case since the publicly available LCs have almost no pre-burst data. This is the main reason why dim bursts were excluded in our sample selection in Sect. 2.

In B04 it was found empirically, considering the analysis of the average ACFs of the narrow and broad width sets and their dispersion, that at half-maximum the separation between the two sets is most significant. Therefore, the ACF half-width at halfmaximum $w$ given by $A(\tau=w) \equiv 0.5$ (here we deviate from the B04 notation for simplicity) was chosen as the measure that best characterises each class. Since it has been shown that the average ACF decreases approximately following a stretch exponential (F95), the ACF width $w$ was calculated fitting the logarithm of the ACF in the range $0.4 \leq A(\tau) \leq 0.6$ with a second degree polynomial.

The uncertainties due to stochastic fluctuation were estimated using a Monte Carlo method. For any given LC a large number of realisations is generated assuming that the fluctuations on the gross number of counts in each bin $m_{i}$ follows a Poisson distribution. Then, the LCs are background subtracted and the ACF widths $w$ are calculated following the same method 

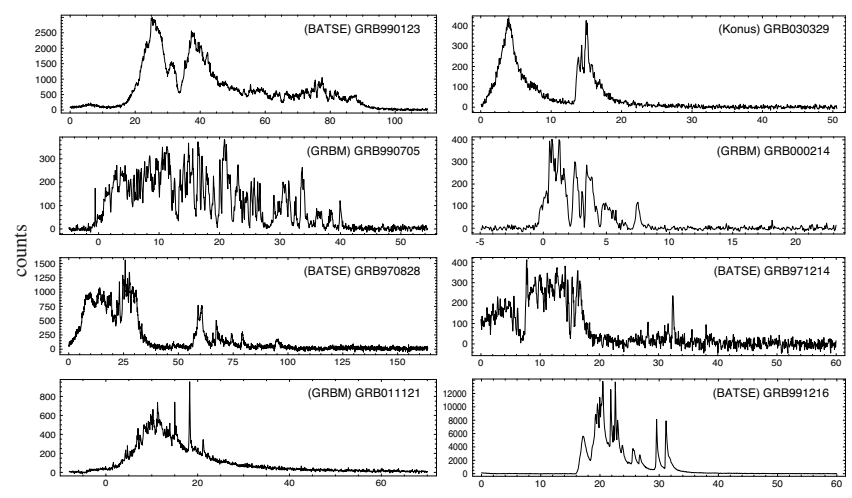

$t^{\prime}[\mathrm{s}]$

Fig. 1. Examples of GRB light curves from the our sample of bursts with known redshifts, where the time $t^{\prime}$ is calculated at the source restframe. The light curves on the left (right) panels have broad (narrow) ACF widths. There are no obvious morphological differences between the two classes, both presenting cases of simple and complex structures.

as for the original data. However, when subtracting the Poisson noise contribution in the ACF (Eq. (2)) a factor of 2 must be introduced in the corresponding noise term to take into account that the procedure to generate the synthetic LCs doubles the noise variance, since they are in a sense second order realisations. In general, if one considers a Poisson process with expected value $\mu$ and iteratively assumes its counts as expected counts of another Poisson process, after $n$ iterations the expected value is still $\mu$, but the variance is $n \mu$ (with $n=2$ in this case). Finally, the standard deviation of the obtained widths is used as estimator of the uncertainty. Nevertheless, the main source of uncertainty in dim bursts is the background estimation, that introduces a systematic error in the determination of the ACF width. Therefore, the reported uncertainties should be regarded as lower limits. Another likely source of systematic errors is the non uniform response of the detectors, since we do not deconvolve the count data.

\section{Analysis of the $\gamma$-ray LCs}

Some LC examples from our sample of GRBs with known redshift are shown in Fig. 1. No morphological differences are evident between the narrow and broad classes by simple inspection. However, standard linear analysis tools reveal clear variability differences between the two, as we report in this section.

\subsection{ACFs}

With the inclusion of GRBM bursts we were able to expand the sample presented in B04. The added ACFs (shown in Fig. 2 in dashed lines) also follows a bimodal distribution when corrected for time dilation effects, reinforcing the previously found pattern. In Fig. 2a the observed ACFs $A(\tau)$ are shown first for comparison, while Fig. 2b shows the rest-frame or local ACFs $A\left(\tau^{\prime}\right)$, where $\tau^{\prime}=\tau /(1+z)$ is the time lag corrected for cosmic dilation. We obtained mean values for the rest-frame ACF half-width at half-maximum ${\overline{w^{\prime}}}_{\gamma}^{(\mathrm{n})}=(1.56 \pm 0.15) \mathrm{s}$ and ${\overline{w^{\prime}}}_{\gamma}^{(\mathrm{b})}=(7.42 \pm 0.14) \mathrm{s}$, and also sample standard deviations $\sigma_{\gamma}^{(\mathrm{n})}=0.6 \mathrm{~s}$ and $\sigma_{\gamma}^{(\mathrm{b})}=0.5 \mathrm{~s}$ for the narrow and broad subsets respectively. The mean values are equal to those reported in B04 within uncertainties. The gap between the two subsets (defined as the mean difference) represents a $4.9 \sigma$ separation, smaller than the $7 \sigma$ separation reported
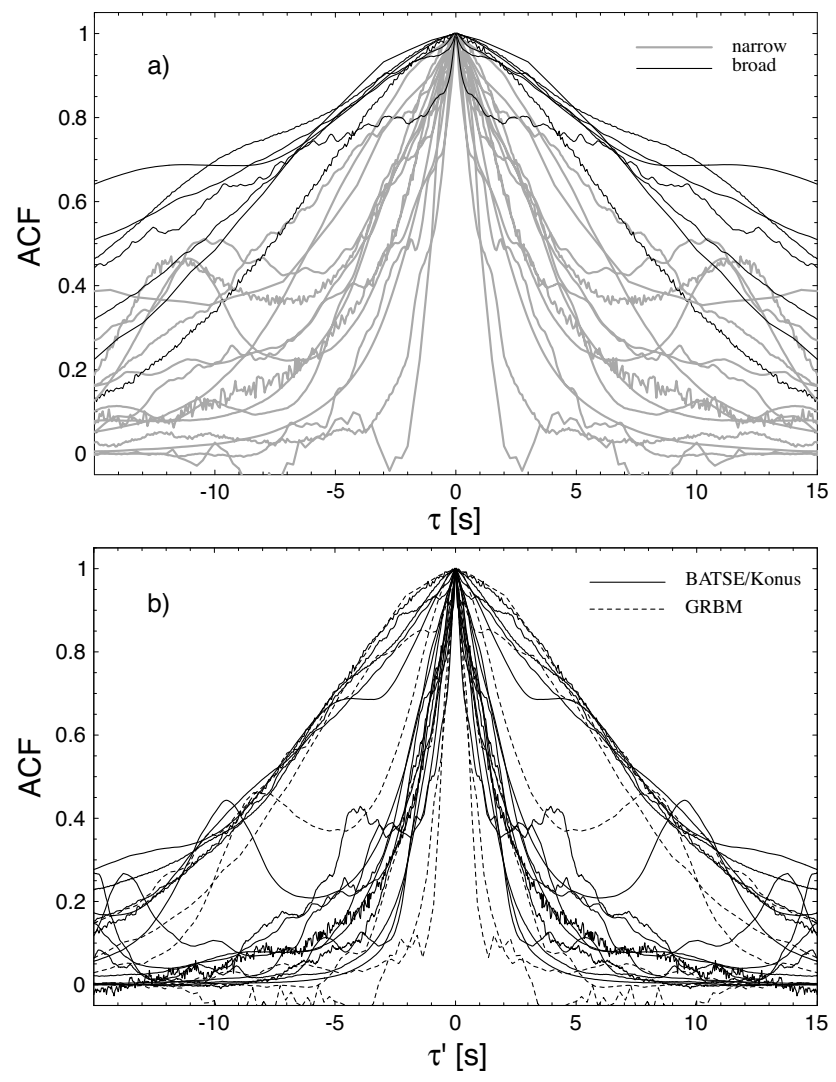

Fig. 2. a) Autocorrelation functions (ACFs) of 22 GRBs with known redshifts calculated in the observer's frame. Although easily identified in the rest-frame, the narrow (gray lines) and the broad (solid lines) classes overlap giving the impression of a unimodal distribution. b) Local ACFs where the cosmic time dilation effect has been corrected, being $\tau^{\prime}=\tau /(1+z)$. The newly added GRBM data (dashed lines) reinforce the bimodal pattern previously found by B04 that used only the BATSE and Konus data (here both shown with solid lines).

in B04 mainly due to the increased dispersion in the broad class. However, thanks to a larger sample, we have slightly increased the significance of this separation. The probability $p$ of a random occurrence of such gap was estimated using Monte Carlo methods. Assuming that there are no characteristic timescales and an underlying uniform probability distribution (the most favourable case) we estimated $p<4 \times 10^{-7}$.

Hereafter our sample of bursts with known redshifts in the $\gamma$ band will be divided for analysis into two subsets of 15 narrow and 7 broad ACF width bursts respectively. In Table 1 we show the newly added ACFs widths together with those presented in B04 for completeness.

\subsection{PDS}

We calculate the PDS for our sample of GRBs with known redshift, correcting the LCs for cosmic time dilation effects. We group bursts following the classes established in Sect. 4.1 based on the ACF width. The upper panels of Figs. 3 and 4 show for the narrow and broad classes all individual PDS overlaid for comparison. Given the chosen normalisation, all PDS must converge to unity at low frequencies (see Sect. 3). The frequencies have been equally binned on the logarithmic scale. The procedure not only smooths out the PDS stochastic variations but enable us to estimate an average PDS for each class. This is necessary because even if all the LCs had the same time duration, the 
Table 1. Sample of 22 GRBs with known redshift. The columns give the name of the GRB, the source instrument for the $\gamma$-ray data, the X-ray instrument when available, the measured redshift $z$, the corresponding reference, the ACF half-width at half-maximum in the $\gamma$-ray energy band $w_{\gamma}$, the half-width corrected for time dilation $w_{\gamma}^{\prime}$, the corresponding half-widths $w_{\mathrm{X}}$ and $w_{\mathrm{X}}^{\prime}$ from the X-ray light curves when available, the width ratio between the two energy bands $w_{\mathrm{X}} / w_{\gamma}$, the index $\xi$ assuming a width energy dependence $w_{\gamma}(E) \propto E^{-\xi}$, and the ACF width class.

\begin{tabular}{|c|c|c|c|c|c|c|c|c|c|c|c|}
\hline GRB & $\begin{array}{l}\text { Instrument }(\gamma) \\
(55-320 \mathrm{keV})^{a}\end{array}$ & $\begin{array}{c}\text { Inst. (X) } \\
(2-28 \mathrm{keV})\end{array}$ & $z$ & $\operatorname{Ref}^{b}$ & $\begin{array}{l}w_{\gamma} \\
(\mathrm{s})\end{array}$ & $\begin{array}{l}w_{\gamma}^{\prime} \\
(\mathrm{s})\end{array}$ & $\begin{array}{l}w_{\mathrm{X}} \\
(\mathrm{s})\end{array}$ & $\begin{array}{l}w_{\mathrm{X}}^{\prime} \\
(\mathrm{s})\end{array}$ & $w_{\mathrm{X}} / w_{\gamma}$ & $\xi_{2 \mathrm{ch}}^{c}$ & Class $^{d}$ \\
\hline 970228 & GRBM & WFC & 0.695 & (1) & $1.3 \pm 0.1$ & $0.77 \pm 0.06$ & $3.5 \pm 0.2$ & $2.1 \pm 0.1$ & 2.7 & & $\mathrm{n}$ \\
\hline 970508 & BATSE/GRBM & WFC & 0.835 & (2) & $2.7 \pm 0.1$ & $1.47 \pm 0.05$ & $10.3 \pm 0.4$ & $5.6 \pm 0.2$ & 3.8 & $0.06 \pm 0.05$ & $\mathrm{n}$ \\
\hline 970828 & BATSE & & 0.9578 & (3) & $15.33 \pm 0.06$ & $7.83 \pm 0.03$ & & & & $0.09 \pm 0.03$ & $\mathrm{~b}$ \\
\hline 971214 & BATSE/GRBM/Konus & WFC & 3.418 & (4) & $8.02 \pm 0.08$ & $1.81 \pm 0.02$ & $11.8 \pm 0.8$ & $2.7 \pm 0.2$ & 1.5 & $0.10 \pm 0.04$ & $\mathrm{n}$ \\
\hline 980326 & GRBM & WFC & 1.2 & (5) & $1.34 \pm 0.1$ & $0.61 \pm 0.04$ & $2.35 \pm 0.4$ & $1.05 \pm 0.2$ & 1.7 & & $\mathrm{n}$ \\
\hline 980329 & BATSE/GRBM/Konus & WFC & $3 \pm 1$ & (6) & $5.96 \pm 0.02$ & $1.5 \pm 0.5$ & $8.8 \pm 0.4$ & $2.2 \pm 0.7$ & 1.5 & $0.05 \pm 0.01$ & $\mathrm{n}$ \\
\hline 980425 & BATSE/GRBM & WFC & 0.0085 & (7) & $7.62 \pm 0.08$ & $7.56 \pm 0.08$ & $14.8 \pm 0.9$ & $14.7 \pm 0.9$ & 1.9 & $0.24 \pm 0.06$ & $\mathrm{~b}$ \\
\hline 980703 & BATSE & & 0.966 & (8) & $14.15 \pm 0.1$ & $7.19 \pm 0.05$ & & & & $0.11 \pm 0.01$ & $\mathrm{~b}$ \\
\hline 990123 & BATSE/GRBM/Konus & WFC & 1.600 & (9) & $19.81 \pm 0.03$ & $7.62 \pm 0.01$ & $35.6 \pm 0.2$ & $13.70 \pm 0.08$ & 1.8 & $0.30 \pm 0.05$ & $\mathrm{~b}$ \\
\hline 990506 & BATSE/GRBM/Konus & & 1.3066 & (10) & $3.83 \pm 0.02$ & $1.66 \pm 0.01$ & & & & $0.20 \pm 0.05$ & $\mathrm{n}$ \\
\hline 990510 & BATSE/GRBM/Konus & & 1.619 & (11) & $2.54 \pm 0.03$ & $0.97 \pm 0.01$ & & & & $0.20 \pm 0.01$ & $\mathrm{n}$ \\
\hline 990705 & GRBM & WFC & 0.86 & (12) & $14.3 \pm 0.2$ & $7.7 \pm 0.1$ & $22.3 \pm 0.4$ & $12.0 \pm 0.2$ & 1.6 & & $\mathrm{~b}$ \\
\hline 990712 & GRBM & WFC & 0.433 & (13) & $4.1 \pm 0.2$ & $2.85 \pm 0.1$ & $4.8 \pm 0.2$ & $3.35 \pm 0.1$ & 1.2 & & $\mathrm{n}$ \\
\hline 991208 & Konus & & 0.7055 & (14) & $3.67 \pm 0.04$ & $2.15 \pm 0.02$ & & & & & $\mathrm{n}$ \\
\hline 991216 & BATSE/GRBM/Konus & & 1.02 & (15) & $3.80 \pm 0.02$ & $1.88 \pm 0.01$ & & & & $0.18 \pm 0.02$ & $\mathrm{n}$ \\
\hline 000131 & BATSE & & 4.500 & (16) & $5.77 \pm 0.08$ & $1.05 \pm 0.01$ & & & & $0.21 \pm 0.06$ & $\mathrm{n}$ \\
\hline 000210 & GRBM/Konus & WFC & 0.846 & (17) & $2.4 \pm 0.2$ & $1.3 \pm 0.1$ & $5.35 \pm 0.3$ & $2.9 \pm 0.2$ & 2.2 & & $\mathrm{n}$ \\
\hline 000214 & GRBM & WFC & 0.47 & (18) & $2.5 \pm 0.4$ & $1.7 \pm 0.3$ & $6.8 \pm 0.3$ & $4.65 \pm 0.2$ & 2.7 & & $\mathrm{n}$ \\
\hline 010222 & GRBM/Konus & WFC & 1.477 & (19) & $3.68 \pm 0.07$ & $1.48 \pm 0.03$ & $42.2 \pm 0.4$ & $17.0 \pm 0.2$ & 11.5 & & $\mathrm{n}$ \\
\hline 010921 & GRBM & & 0.451 & (20) & $9.8 \pm 0.3$ & $6.75 \pm 0.2$ & & & & & $\mathrm{~b}$ \\
\hline 011121 & GRBM/Konus & WFC & 0.362 & (21) & $10.0 \pm 0.3$ & $7.35 \pm 0.2$ & $18.9 \pm 0.2$ & $13.9 \pm 0.15$ & 1.9 & & $\mathrm{~b}$ \\
\hline 030329 & Konus & & 0.1685 & (22) & $2.6 \pm 0.1$ & $2.19 \pm 0.08$ & & & & & $\mathrm{n}$ \\
\hline
\end{tabular}

${ }^{a}$ The energy range of BATSE data which was taken as reference instrument. For Konus and GRBM data the actual energy ranges are 50-200 keV and 40-700 keV respectively. ${ }^{b}$ (1) Bloom et al. (2001); (2) Metzger et al. (1997); (3) Djorgovski et al. (2001); (4) Kulkarni et al. (1998); (5) Bloom et al. (1999); (6) Lamb et al. (1999); (7) Tinney et al. (1998); (8) Djorgovski et al. (1998); (9) Kulkarni et al. (1999); (10) Bloom et al. (2003); (11) Beuermann et al. (1999); (12) Amati et al. (2000); (13) Vreeswijk et al. (2001); (14) Dodonov et al. (1999); (15) Vreeswijk et al. (1999); (16) Andersen et al. (2000); (17) Piro et al. (2002); (18) Antonelli et al. (2000); (19) Jha et al. (2001); (20) Price et al. (2002); (21) Garnavich et al. (2003); (22) Greiner et al. (2003). ${ }^{c}$ Index $\xi$ has been estimated for an energy window width of two BATSE channels. ${ }^{d}$ (n) and (b) indicate narrow and broad width ACF class, respectively.

redshift correction would make the Fourier frequencies differ for each burst (i.e., since the observed frequency $f$ transforms to the rest-frame as $f^{\prime}=f(1+z)$ ). The lower panels of Figs. 3 and 4 show the corresponding central values $\tilde{P}_{f}$, where we have chosen the use of the median over the mean as a more robust estimator of the expected or "underlying" spectrum. Although qualitatively similar results are obtained in both cases, the median shows smaller fluctuations and it is less sensitive to the random exclusion of a few bursts from each class. The figures show also the quartile deviation (dashed lines) as a measure of the dispersion that was chosen to be consistent with the use of the median (i.e., the $25 \%$ and the $75 \%$ quartiles around the median value). Comparing the two median PDS it is evident that they have remarkably different shapes. The narrow class PDS as expected shows more power at high frequencies and it is well described by a single power-law model $P_{f} \propto 1 / f^{\alpha}$ up to a low frequency cut-off at $\approx 0.1 \mathrm{~Hz}$. In Fig. $3 \mathrm{~b}$ we show a fit to the median PDS in the frequency range $0.1 \leq f^{\prime} \leq 10 \mathrm{~Hz}$ for which we found a best-fit-parameter $\alpha_{\gamma}^{(\mathrm{n})}=1.97 \pm 0.04$ (i.e., $\gamma$ band - narrow class). The obtained exponent is consistent with that of Brownian or red noise (i.e., with $\alpha=2$ ), which suggests the alternative use of a single Lorentzian model to describe the entire PDS as in shot noise models (Belli 1992). However, as shown also in Fig. $3 b$, there seems to be a slight systematic deviation around the cut-off region. The broad class $\widetilde{P}_{f}$ on the other hand appears to have two distinct components, i.e., a broad low frequency component with a sharp break and a power-law component (Fig. 4b). If we assume that the two components are independent then the single power-law should have an independent cut-off at low frequencies which cannot be determined but only constrained by our data. The low frequency component is well fitted by a stretched exponential $P_{1}(f)=\exp \left[\left(-f / f_{1}\right)^{\eta}\right]$. The best-fit-parameters for this model will depend to some extent on the cut-off frequency parameter of the power-law component and vice versa, however this will affect mainly the estimation of the exponent $\eta$. Taking into account these uncertainties we found a characteristic frequency $f_{1}=(0.025 \pm 0.003) \mathrm{Hz}$ and an index $\eta=1.3 \pm 0.2$. We considered other empirical models, concluding that functions with power-law asymptotic behaviour (e.g., a Lorentzian) do not decay fast enough to fit the data. For the high frequency component we found a best-fit power-law index $\alpha_{\gamma}^{(\mathrm{b})}=1.6 \pm 0.2$ (i.e., $\gamma$ band - broad class), consistent with the Kolmogorov spectral value $5 / 3$. However, note from Fig. 4a that for different bursts the latter component appears to vary much more than the first.

As we discussed in Sect. 1, the obtained mean PDS (as well as the median) may depend on the chosen normalisation since each burst will be weighted in a different way. If all LCs in the sample are realisations of the same stochastic process then the norm should simply improve the convergence to the mean value but should give identical results for a sufficiently large sample. Aside from the fluence norm, we tested several ways to scale the LCs, e.g., the peak flux used previously by Beloborodov et al. (1998, 2000), the Miyamoto et al. (1992) normalisation (which 


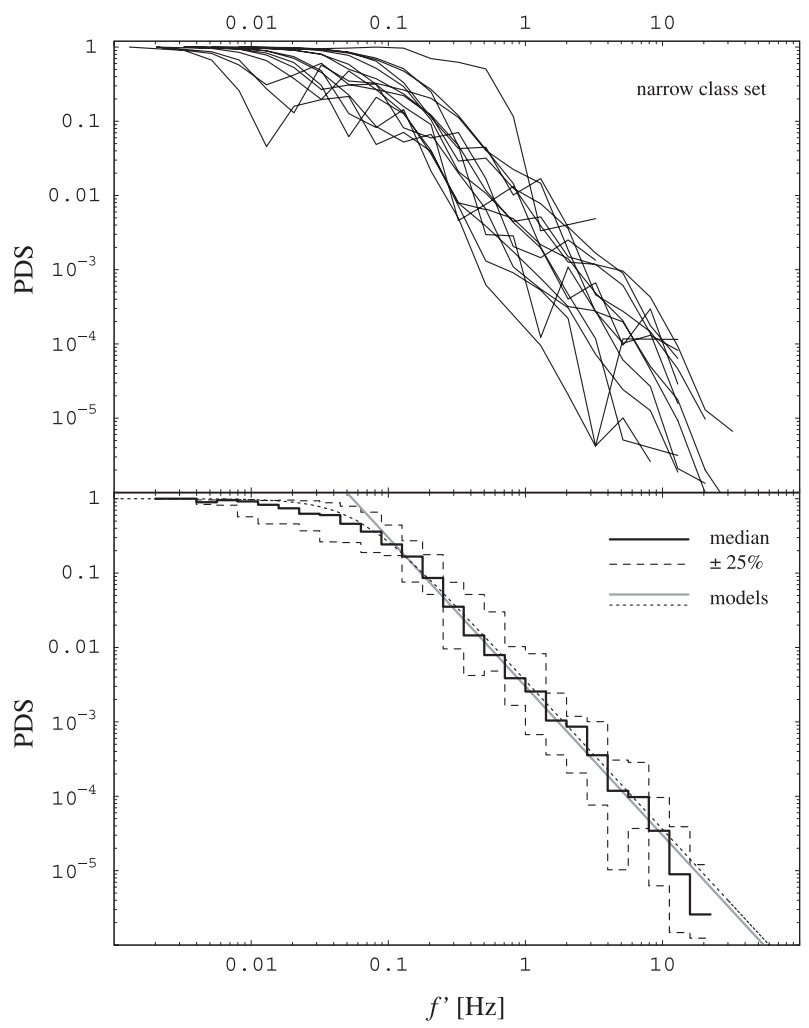

Fig. 3. Power density spectra (PDS) of the subset of 15 bursts with narrow width ACFs. The frequency scale has been corrected for cosmic time dilation effects and the noise level has been subtracted assuming Poisson statistics. The upper panel shows all individual PDS where the frequency data have been equally binned in the logarithmic scale. The lower panel shows the estimated sample median (solid line) and the quartiles about the median (dashed lines) to indicate the dispersion. The decay phase is well modelled by a power-law (gray line) with index $1.97 \pm 0.04$. Also shown, a Lorentzian function (dotted line) provides a fairly good fit over the whole frequency range.

expresses the PDS in fractional root-mean-square), and the root of the total power $\sqrt{P_{\text {tot }}}$ used for the ACF. We found in all cases qualitatively the same median PDS for each class but significantly larger spreads. Obviously, this does not prove that each class sample is "uniform" since it is a necessary but not sufficient condition. In this respect, it is worth mentioning that when combined into a single sample and without correcting for redshifts effects we obtained a median PDS (shown in Fig. 5) that is well fitted in the $0.01-1 \mathrm{~Hz}$ frequency range by a single power-law with index $1.73 \pm 0.07$, consistent within uncertainties with the Kolmogorov spectrum and in full agreement with Beloborodov et al. (1998). Therefore, our sample of GRBs with known $z$ is not different, at least in this regard, to the general sample of bright BATSE GRBs used in previous works. Note also that the overall dispersion about the median is significantly larger than in Figs. 3 and 4 where, despite the smaller sample sizes, redshift corrections and ACF classes have been taken into account.

\section{Analysis of the X-ray LCs}

In this section we use the WFC sample of 13 GRBs with known redshifts (see Table 1) to extend the previous temporal analysis into the X-ray energy range. These bursts represent a subset of the previously used sample in the $\gamma$-ray band (Sect. 4). We find also at these energies significant differences between

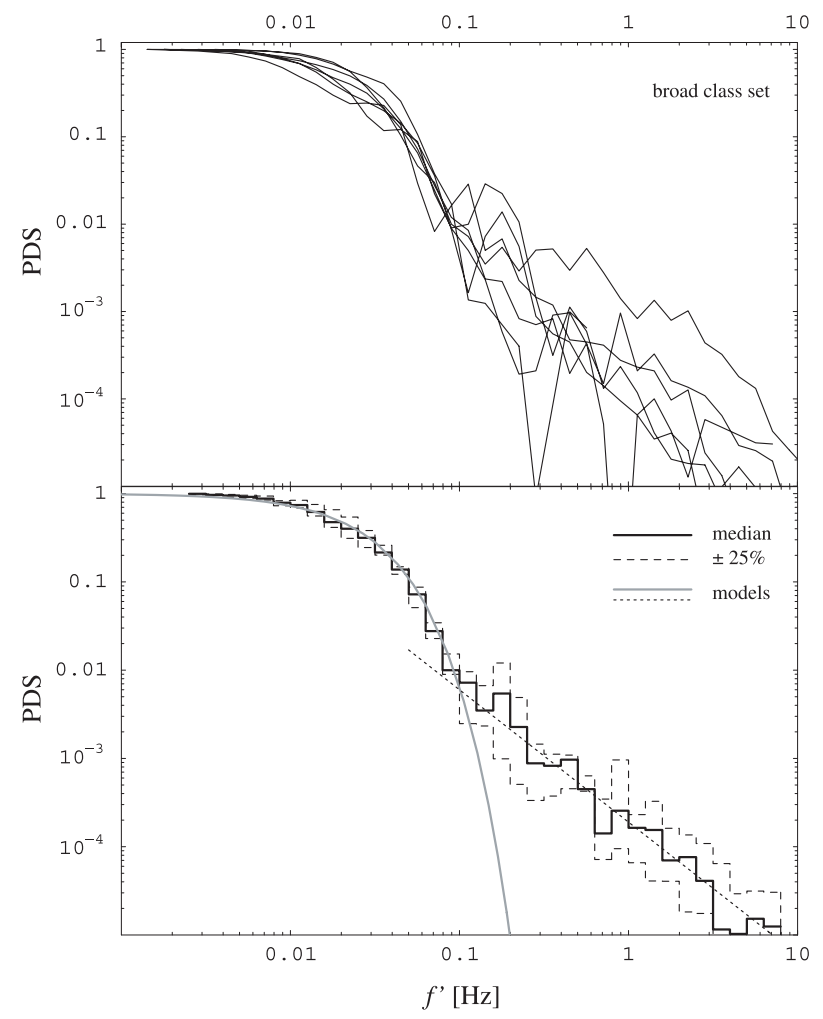

Fig. 4. Power density spectra (PDS) of the subset of 7 bursts showing broad width autocorrelation functions (ACFs). As in Fig. 3 the individual PDS and the sample median are shown for comparison. The median PDS is significantly different to that of the narrow class and two components appear to be present. The low frequency component has a sharp cut-off and is well modelled by a stretched exponential (gray line). The high frequency component is well described by a power-law with index $1.6 \pm 0.2$ consistent with a $5 / 3$ Kolmogorov spectral index.

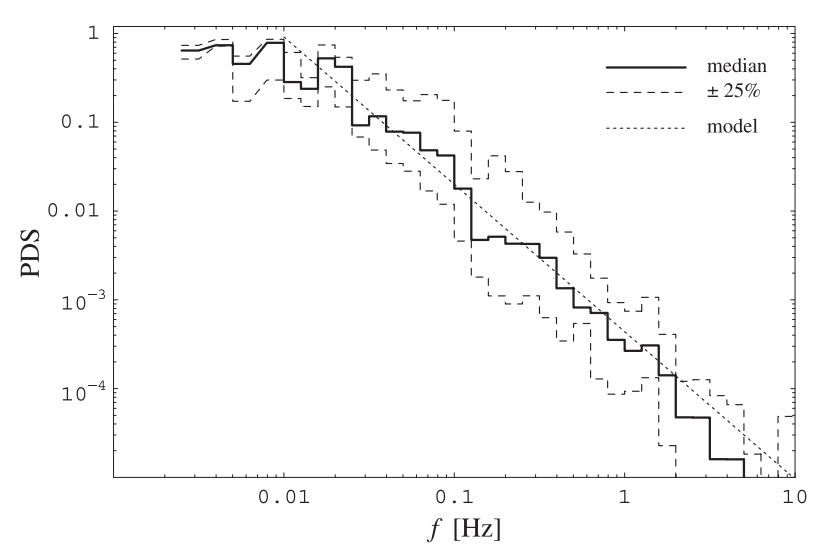

Fig. 5. Median power density spectra of our whole sample of 22 GRBs with known redshift calculated in the observer's frame. The spectrum shows a power-law behaviour approximately within the $0.01-1 \mathrm{~Hz}$ frequency range and a best fit (dotted line) gives an index $1.73 \pm 0.07$, consistent with the results of Beloborodov et al. (1998) derived without redshift corrections. Note that the quartile dispersion (dashed lines) is considerably larger than those found in Figs. 3 and 4.

the two ACF classes although the results inevitable have larger associated uncertainties. 


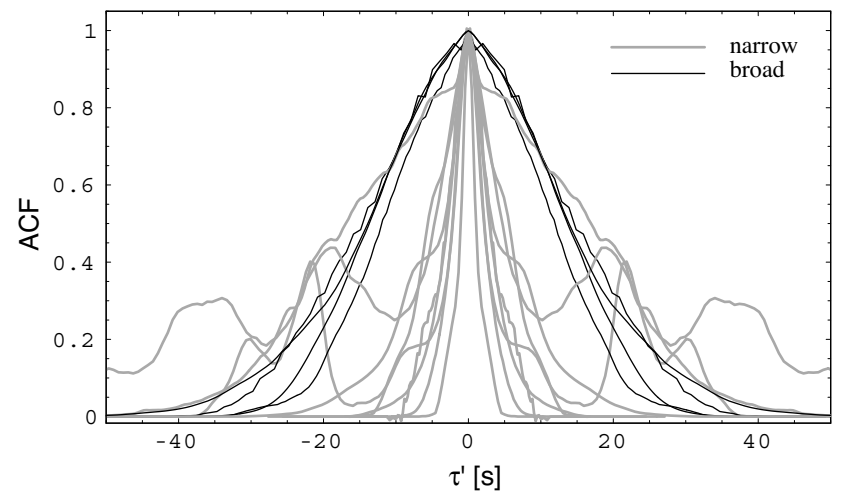

Fig. 6. Autocorrelation functions (ACFs) calculated at the source restframe for a sample of 13 GRBs detected by WFC in the $2-28 \mathrm{keV}$ energy band. In spite of the broadening of the ACF at lower energies, the bimodal distribution found in the $\gamma$-ray band is clearly seen, although with larger relative dispersion for each class. However, one burst (GRB 010222) previously classified as belonging to the narrow ACF width class (gray lines) appears wider than the broad ACF width class (solid lines). This behaviour is most likely not intrinsic but due to a systematic error (see discussion in Sect. 7).

\subsection{ACFs}

Although the ACF widths are substantially broader at these lower energies (2-26 keV), the two ACF classes are still easily distinguishable as shown in Fig. 6. However, the GRB 010222 classified as having a narrow ACF in the $\gamma$ band (gray lines) has broaded to such extent that it falls into the broad width range, although its ACF decays more slowly than any of the broad width cases. As shown in Table 1 where we list all the observed and the local ACF widths, this is the only outlier in the sample. In Col. 10 we list as a measure of the broadening the ACF width ratio for the $\gamma$ and $\mathrm{X}$-ray bands. While the average width ratio is $\left\langle w_{\mathrm{X}} / w_{\gamma}\right\rangle \simeq 2$, the broadening in the GRB 010222 case $\left(w_{\mathrm{X}} / w_{\gamma} \simeq 11.5\right)$ is much larger than any other burst in the sample and it is most likely caused by a systematic error, as we will discuss later in Sect. 7. For this reason this case will be kept separate from the sample for the rest of our WFC data temporal analysis. Consequently, the obtained average local width ACF are ${\overline{w^{\prime}}}_{\mathrm{X}}^{(\mathrm{n})}=(3.1 \pm 0.5) \mathrm{s}$ and $\bar{w}_{\mathrm{X}}^{\prime(\mathrm{b})}=(13.6 \pm 0.6) \mathrm{s}$, with sample standard deviations (relative dispersions) $\sigma_{\mathrm{X}}^{(\mathrm{n})}=1.9 \mathrm{~s}(60 \%)$ and $\sigma_{\mathrm{X}}^{(\mathrm{b})}=1.6 \mathrm{~s}(12 \%)$ for the narrow and broad subsets respectively.

\subsection{PDS}

Once again using our sample of WFC bursts with known $z$, we estimate the local median PDS for each ACF width class following the same methods used in Sect. 4.2. We consider separately the special case GRB 010222 due to the unique broadening of its ACF. Figure 7 shows our results for the narrow class. As expected from the ACF analysis the break appears now at approximately half the corresponding frequency at $\gamma$ energies (Fig. 3), but most noticeably the behaviour towards the high frequencies now shows a much faster decay. A best-fit for frequencies $f^{\prime} \gtrsim 0.08 \mathrm{~Hz}$ using a $P_{f} \propto 1 / f^{\alpha}$ model gives an exponent $\alpha_{\mathrm{x}}^{(\mathrm{n})}=3.0 \pm 0.2$, a significantly steeper power-law than in the $\gamma$ band. The PDS of GRB 010222 (gray line) is also shown in Fig. 7 for comparison. Although with much less power at low frequencies than the other bursts in this class, at high frequencies $\left(f^{\prime} \geq 0.2 \mathrm{~Hz}\right)$ it follows a very similar asymptotic behaviour.

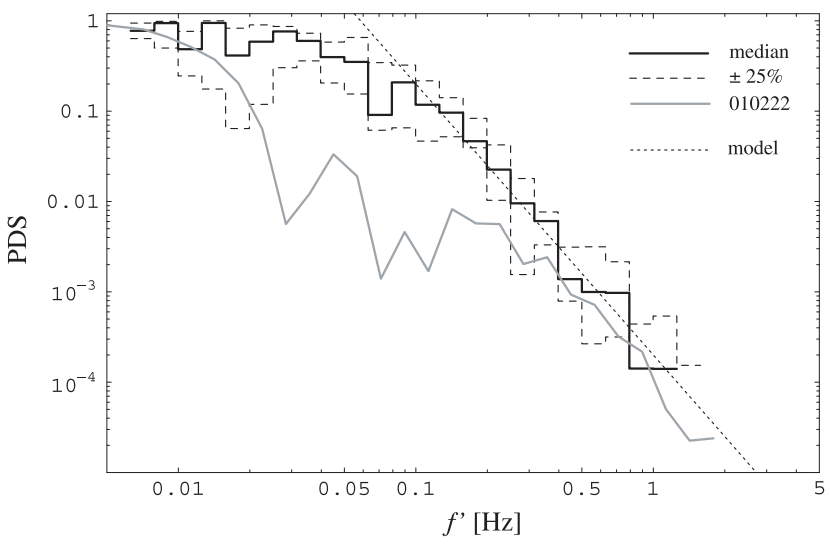

Fig. 7. Median power density spectra (PDS) from the subset of WFC bursts with known redshifts belonging to the narrow width ACFs class, excluding the outlier case GRB 010222 that is shown along side (gray line $)$. The decay at high frequencies $\left(f^{\prime}>0.1 \mathrm{~Hz}\right)$ approximately follows a power-law, but with a significantly steeper slope than the previously found in the $\gamma$-ray energy band $(3.0 \pm 0.2)$. GRB 010222 shows similar asymptotic behaviour at high frequencies.

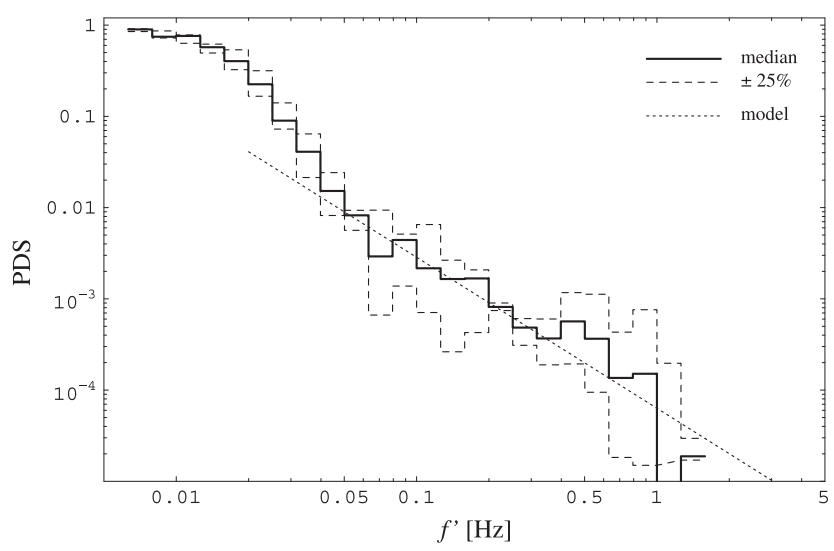

Fig. 8. Median power density spectra (PDS) from the subset of 4 WFC bursts with known redshifts belonging to the broad width ACFs class. Despite the small sample size, the two broad PDS components identified at $\gamma$-ray energies are recognisable. The index of the power-law component (dotted line) is consistent with the Kolmogorov value as previously found in Fig. 4 given a best-fit-parameter $1.7 \pm 0.2$.

Our estimation of the median PDS $\tilde{P}_{f}$ for the broad class is shown in Fig. 8. In spite of the large uncertainties associated with such a small sample, the two components found in Fig. 4 are recognisable. The low frequency component is not as prominent and broad as before, and consequently the data are not enough to properly constrain the fit parameters of a stretched exponential as it was previously done. However, we estimated that the high frequency component follows a power-law with index $\alpha_{\mathrm{X}}^{(\mathrm{b})}=1.7 \pm 0.2$ consistent within uncertainties with the Kolmogorov index found in Sect. 4.2 for the $\gamma$-ray LCs. When compared with the broad class $\tilde{P}_{f}$ in Fig. 8, the GRB 010222 spectrum matches neither the $\tilde{P}_{f}$ general shape nor any of its components. Power-law fits using the indices and the frequency ranges shown in Figs. 7 and 8 for the respective high-frequency components resulted in squared residual totals per degrees of freedom $\chi^{2} / v=16.2 / 11$ and $\chi^{2} / v=179 . / 17$ for the narrow and broad PDS respectively. Based on these fits we conclude that the first model is acceptable while the second model can be rejected at a very high confidence level. Uncertainties were estimated using synthetic shot noise and calculating standard deviations on 
a sample of PDS following the same procedures and frequency binnings applied to the burst data.

\section{Comparing ACF classes}

In order to understand the origin of the two classes found based on our temporal analysis we looked for any additional GRB characteristic or physical parameter that might differ between them. As point out in Sect. 4, the visual inspection of the LCs reveals no trivial morphological differences between the classes (see Fig. 1). Both classes present cases with simple and complex structure, showing from a few smooth pulses to many heavily overlapped sharp pulses. To cover a broad range of physical parameters, we made use of the database of GRB redshifts and other burst parameters compiled in Friedman \& Bloom (2005) and references therein, that contains much additional information about the 22 bursts in Table 1, although not all parameter estimations are available for every burst. In particular, we considered the following parameters (primed quantities are calculated at the source rest-frame): the peak energy $E_{\mathrm{pk}}^{\prime}$, the isotropic equivalent gamma-ray energy $E_{\mathrm{iso}}$, the duration time $T_{90}^{\prime}$, the time of the observed break in the afterglow light curves $T_{\mathrm{b}}^{\prime}$, and the redshift $z$. In all cases we compared the distributions within the narrow and broad ACF classes using the standard Kolmogorov-Smirnov (K-S) test (see, e.g., Press et al. 1992), which is particularly sensitive to median deviations, but we found no significant difference for the cumulative distributions of any of those parameters. No significant $R$ correlation coefficients with $w^{\prime}$ were found for $E_{\mathrm{pk}}^{\prime}$ and $E_{\text {iso }}$ (logarithms taken in all cases), although marginal results were obtained for the temporal parameters $T_{90}^{\prime}(R=0.44$ with significance $p<0.04)$ and $T_{\mathrm{b}}^{\prime}(R=0.46$ with significance $p<0.05$ for only 18 bursts with determined values). Note that a correlation between $w$ and $T_{90}$ has been well established $(R=0.58)$, and although in the observer-frame is partially due to the cosmic temporal dilations, it should be found also in the rest-frame (Borgonovo \& Björnsson 2006). However, our present sample is too small to determine it at a high confidence level. More surprising is the finding of an indication of some correlation between a prompt $\left(w^{\prime}\right)$ and an afterglow $\left(T_{\mathrm{b}}^{\prime}\right)$ parameter, but this would need better statistics to be confirmed.

Other subdivisions of the long/soft GRB class have been suggested in the literature but we found them to be unrelated to with the one under consideration. Evaluation of the distributions of the observed $T_{90}$ and $E_{\mathrm{pk}}$ for the broad ACF class excludes an association between these bursts and the "intermediate" duration GRB group reported by Horváth (1998) and Mukherjee et al. (1998). Likewise, we found no correspondence between the ACF bimodality and the bimodality of the afterglow optical luminosity distribution reported by Nardini et al. (2006) and Liang \& Zhang (2006).

\section{ACF energy dependence}

In our analysis we have only corrected the LCs for cosmic time dilation. However, the data are collected over a finite energy band and therefore additional corrections might be needed to account for the shift in energy, since in principle we should compare LCs emitted in the same energy band. As LC pulses are sharper at higher energies (Norris et al. 1996), correspondingly the ACF is narrower. For a sample of 45 bright bursts, F95 found that the width of the mean ACF depends on the energy $E$ as $w(E) \propto E^{-0.43}$. Since for large redshifts the instrument will see photons emitted at higher mean energies, the anti-correlation of the ACF width with the energy should partially counteract the time dilation effect. In B04 possible additional redshift corrections to the accounted cosmic dilation were studied using the transformation

$w^{\prime}=w /(1+z)^{1+a}$

for the observed widths, where $a$ represents a smaller than unity correction. The relative dispersion of each width class was calculated as a function of the parameter $a$ looking for minimum values (see Fig. 6 in B04) since, under the assumption that the $w^{\prime}$ intrinsic distribution does not depend on $z$ (i.e., evolution effects are neglected), redshift dependencies should increase the observed dispersion. We obtained minimal dispersions at $a_{\text {min }}^{(\mathrm{n})}=-0.35 \pm 0.2$ and $a_{\text {min }}^{(\mathrm{b})}=0.04 \pm 0.04$ for the narrow and broad classes respectively, equal within uncertainties to previous results. The uncertainties were estimated numerically using the bootstrap method (see, e.g., Press et al. 1992). If the dispersion is calculated over the whole sample (i.e., without separating the two width classes) no well-defined minimum is found, with an approximately constant value within the free parameter range $|a|<1$, which further supports the separation into two classes. However, while the narrow class minimum agrees within errors with the expected effect from the energy shift, the broad class dispersion does not improve much with any additional redshift correction. Actually, in both cases a perfect cancellation (i.e., $a_{\min }=0$ ) cannot be ruled out, although it would appear coincidental that all other possible redshift dependencies would exactly compensate the energy shift. Another possibility is that for the broad class the ACF dependence with energy is weaker. At face value the low dispersion already suggests this and in this case a nearly null $a$ correction would be much more likely. In what follows we will show evidence that supports the latter alternative.

Using 4 energy channel data from a sample of 188 bright BATSE bursts we analyse the ACF width dependence with energy for each individual case using a single power-law model $w(E) \propto E^{-\xi}$ (the channels are approximately equally spaced on a logarithmic scale). We found that in most cases the relation $w(E)$ in individual bursts is not well fitted by a powerlaw (less than $33 \%$ of the bursts satisfied for the sum of the reduced residuals $\chi_{\mathrm{r}}^{2}<2$ ), but for most cases it can be considered a good approximation within the BATSE energy range with typical total relative errors $<10 \%$. The obtained distribution of $\xi$ indices is shown in Fig. 9. Since we found for our fits poorer figures-of-merit (i.e., $\chi_{\mathrm{r}}^{2}$ ) for larger $\xi$ values, the largest values are related to the cases that strongly deviate from the power-law model for $w(E)$ and are therefore ill determined. For this same sample, a power-law fit of the energy dependence of the width of the mean ACF gives an index $-0.40 \pm 0.03$ in agreement with F95. However, note in Fig. 9 that for such a long-tailed distribution most individual bursts typically have a weaker energy dependence and therefore the median $\widetilde{\xi}_{1 \mathrm{ch}}=0.29$ is a more representative value of individual indices.

In addition, since we are particularly interested in quantifying the effect of the energy dependence in our ACF analysis where the observation energy window has the width of two BATSE channels, we studied the ACF width energy dependence $w(E)$ combining the 4-channel data into three overlapped 2-channel width energy bands. The distribution of the thus obtained indices $\xi_{2 \mathrm{ch}}$ is shown in Fig. 9 (dashed lines). The median $\tilde{\xi}_{2 \mathrm{ch}}=0.21$ is significantly smaller, as expected since less correction will be needed the wider the energy window. If we consider the median value of the ACF broadening $w_{\mathrm{X}} / w_{\gamma}$ for the sample 


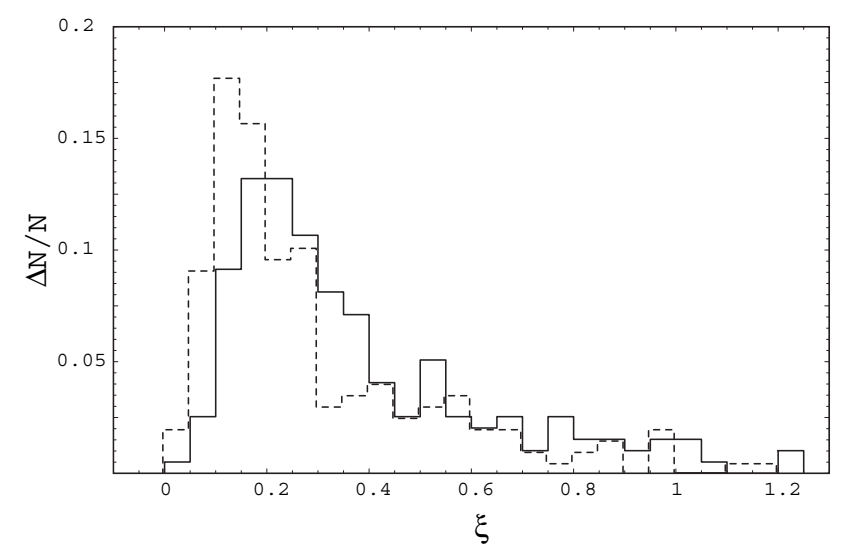

Fig. 9. Distribution of the index $\xi$ in the power-law relation $w(E) \propto E^{-\xi}$ used to model the energy dependence of the ACF width, obtained using 4-channel concatenated data (solid line) for a sample of 188 bright BATSE bursts. The distribution has an estimated median $\tilde{\xi}_{1 \mathrm{ch}}=0.29$ and it shows an approximately exponential decay towards larger $\xi$ values. For the purposes of our ACF analysis that uses the two central BATSE channels, we modelled also the $w(E)$ relation using a 2-channel energy window for each data point. As expected, the ACF width shows a weaker energy dependence and consequently lower typical values for the $\xi$ index. The thus obtained distribution (dashed line) is narrower and with a significantly lower median $\tilde{\xi}_{2 \mathrm{ch}}=0.21$.

in Table 1 (excluding GRB 010222) we derive a median index $\tilde{\xi} \approx 0.20$, in good agreement with the 2 -channel energy window distribution in Fig. 9, either taking the lower edge of each energy window (following F95) or their geometric means. In Table 1 we list our estimations of the index $\xi_{2 \mathrm{ch}}$ for the 11 BATSE GRBs with known $z$. Both ACF width classes show typical values that seem consistent with the general distribution in Fig. 9, but unfortunately the small sample size only allowed us to distinguish very substantial differences.

Since we do not have redshifts for most of the BATSE GRBs, the estimated distribution of index $\xi$ was derived mixing both ACF width classes; nevertheless it is probably fairly representative of the narrow class that dominates the sample. In principle, we do not know how representative that general distribution is of the broad class. For our sample of 11 BATSE GRBs with known $z$ the fraction of broad cases is $f_{\mathrm{b}}=4 / 11\left(f_{\mathrm{b}}=7 / 22\right.$ for our whole sample). However, since the afterglow localisations needed for the redshift determinations always involved another instruments with different trigger responses, that fraction is most likely not representative of the general BATSE catalog. An analysis of the ACF widths of the 188 brightest long BATSE bursts sample shows that the distribution is well described by a $\log$-normal distribution with a mean $10^{\langle\log w\rangle}=3.0 \mathrm{~s}$ and a standard deviation representing a factor of 3.1, with no significant hint of an underlying bimodality (see also Borgonovo \& Björnsson 2006). Since all 11 BATSE GRB with known redshift have $w>2.5 \mathrm{~s}$ almost half of the BATSE $w$ distribution without redshifts seems truncated. Using the K-S test that compares cumulative distributions we calculate a chance probability $p<0.02$ that they are drawn from the same distribution. We conclude that the sample with known $z$ is significantly biased towards large ACF widths (in agreement with Muñoz \& Tan 2005), mainly affecting the representation of the narrow class. Taking into account this truncation and the fact that for a burst to belong to the broad class its observed ACF width must be $w \gtrsim 7 \mathrm{~s}$ independently of the redshift, we estimate that the unbiased BATSE fraction of broad cases should lie

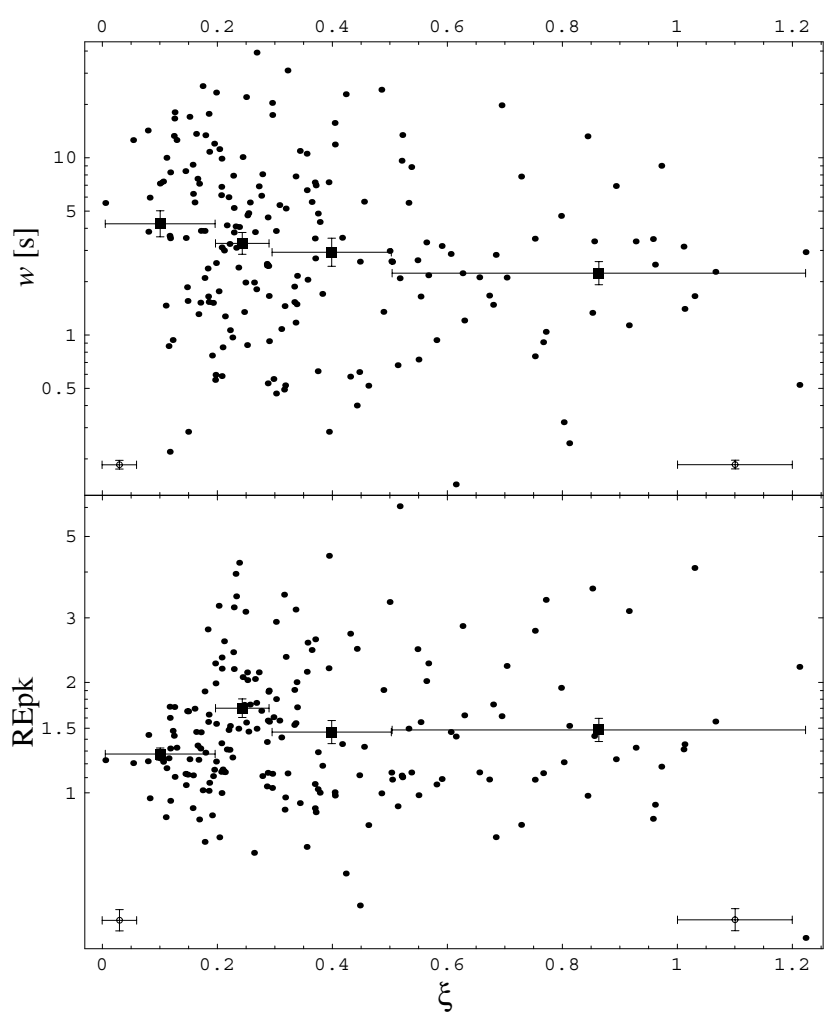

Fig. 10. Correlation analysis for the index $\xi$ from the relation $w(E) \propto$ $E^{-\xi}$ describing the energy dependence of the ACF width based on a sample of 188 BATSE bursts. Although we found no overall correlation between $\xi$ and other commonly used burst parameters (see Sect. 7), at small $\xi \lesssim 0.3$ values two parameters show a significantly different behaviour comprising $\sim 50 \%$ of the sample. At the lower corners we show average values of the estimated uncertainties representative of each data halves. Large $\xi$ values have much larger uncertainties and they are usually associated with poor fits. The $\xi$ axis has been divided into four bins with equal number of data points for averaging (solid squares). Upper panel: typical values of the ACF width $w$ are higher for lower $\xi$ indices. Lower panel: the ratio of peak energies $\mathcal{R} E_{\mathrm{pk}}$, that is a measure of the burst energy spectral evolution, shows less dispersion and a positive correlation at low $\xi$ indices.

approximately between $0.08 \lesssim f_{\mathrm{b}} \lesssim 0.22$ to be consistent with the observations.

Having characterised the energy dependence of the timescale $w$ with the index $\xi$, we investigated possible correlations with other GRB parameters, but bearing in mind that the largest $\xi$ values are most likely meaningless. We tested a broad range of temporal and spectral parameters (e.g., duration time $T_{90}$, emission time, ACF width $w$, time lag between energy channels, $v F_{v}$ peak energy $E_{\mathrm{pk}}$, low and high spectral indices $\alpha$ and $\beta$ ) following definitions and methods described in Borgonovo \& Björnsson (2006) for a multivariate correlation analysis. In particular, we used a measure of the overall spectral evolution of a burst introduced there, where the LC is divided in two fluence-halves and the peak energies of the corresponding integrated spectra are estimated and compared. In that way a ratio of peak energies is defined as $\mathcal{R} E_{\mathrm{pk}} \equiv E_{\mathrm{pk}}{ }^{(1)} / E_{\mathrm{pk}}{ }^{(2)}$, so that $\mathcal{R} E_{\mathrm{pk}}>1$ implies an overall hard to soft evolution. Although we found no significant global correlations with the index $\xi$, there are indications of a different behaviour at low $\xi$ values in two of the studied parameters, i.e., $w$ and $\mathcal{R} E_{\mathrm{pk}}$.

In Fig. 10 we show scatter plots of the ACF width $w$ and the ratio of peak energies $\mathcal{R} E_{\mathrm{pk}}$ versus the index $\xi$. The $\xi$ observed 
range was divided into four bins with equal number of data points, and the corresponding geometric means (more appropriate for log-normal distributions) and uncertainties are shown. Due to the large spread the variables seem uncorrelated based on their correlation coefficients $R$, nevertheless the binning reveals a trend where lower $\xi$ values correspond on average to broader $\mathrm{ACF}$ widths $w$ and lower values of the $\mathcal{R} E_{\mathrm{pk}}$ ratio. In addition, a weak but significant anti-correlation exists between $w$ and $\mathcal{R} E_{\mathrm{pk}}$ with coefficient $R=-0.25$ taken their logarithms. For $\xi \lesssim 0.3$ there is noticeably less dispersion in $\log \mathcal{R} E_{\mathrm{pk}}$ (i.e., less relative dispersion in $\mathcal{R} E_{\mathrm{pk}}$ ) and a clear positive correlation that comprises half of the sample (with coefficient $R=0.45$ ). Comparing a linear fit to those data to the no correlation null hypothesis gives for the F-test (the standard test for a ratio of $\chi^{2}$ values) a level of rejection $p<0.001$. However, since we do not have an objective criterion to truncate the index $\xi$ distribution, the real significance of this found correlation is hard to evaluate; therefore we approach the problem using Monte Carlo methods. We draw random samples for both parameters using the bootstrap method so that they are uncorrelated. Then sorting the data based on their $\xi$ values, we look for the $\xi$-truncate that would give the maximum chance correlation with $\mathcal{R} E_{\mathrm{pk}}$. Assuming conservatively that the correlation has to comprise at least $1 / 3$ of the sample and $|R|>0.3$ then the trial is judged as successful. In this way we found a probability of $p<0.008$ of finding a similar or stronger correlation by chance. Although the energy dependence of the ACF is clearly a consequence of the spectral variability, the spectral evolution of a burst is as complex as its LC, shifting from hard to soft over each individual pulse (Borgonovo \& Ryde 2001). For this reason it is only under certain limited conditions that we are able to actually observe a correlation between $\xi$ and $\mathcal{R} E_{\mathrm{pk}}$. In summary, from the analysis of Fig. 10 there are clear indications that bursts with broad ACF on average show weaker ACF energy dependence and less spectral evolution.

Finally, we attempted to extend the analysis of the ACF energy dependence to X-ray energies with the use of 3-channel data from the WFC bursts which were simultaneously observed by BATSE. However, only the signal of the very brightest of the WFC bursts were found suitable for this purpose and the resultant sample was considered too small for a systematic broad-band analysis. Furthermore, although for most of these bursts $w(E)$ shows a smooth, approximately power-law behaviour, we observed in a significant number of bursts a function discontinuity or "shift" between the WFC and BATSE data, a systematic error most likely due to the background estimation. The observed fraction of cases that present this artifact ( $\$ 20 \%)$ is consistent with the occurrence of one such case within the 13 WFC bursts with known $z$ in Table 1. Therefore, we conclude that the unusual ACF broadening of the outlier case GRB 010222 discussed in Sect. 5.1 is most likely an artifact. The PDS analysis also supports this since an incorrect background subtraction would mainly affect the power at low frequencies, and we have shown in Fig. 7 that GRB 010222 deviates from the narrow class typical behaviour only at those frequencies.

\section{Discussion}

The reported ACF bimodality in B04 was based on the analysis of a small sample of GRBs with available $z$. Nevertheless, the gap between the two ACF classes was found to be highly significant (with $p<6 \times 10^{-7}$ ). Low-number statistics can often suggest nonexistent features and should be regarded with caution. As always, one should consider the possibility of a selection effect not accounted for in the probability estimation. However, while it is easy to conceive of upper and lower biases in the observed ACF width distribution (e.g., due to trigger criteria), it is harder to think of a reason why this would happen for middle range $w^{\prime}$ values. We have shown in Sect. 7 that, when compared to a complete, flux limited large sample of BATSE bursts, our sample with known $z$ is likely biased against very narrow ACFs. The addition of GRBM data with their larger intrinsic dispersion only slightly increased the statistical significance of the found ACF bimodality. Nevertheless, with three sets of $\gamma$-ray data showing the same bimodal pattern (Fig. 2) we can safely rule out an instrumental effect as the cause of it.

It is clear from the PDS analysis presented in Sects. 4 and 5 that narrow and broad ACF bursts have very different overall variability, with the latter showing a prominent low-frequency PDS component with a very sharp cut-off, which explains in part the associated low dispersion observed in this ACF width class, despite the typical fluctuations in individual burst PDS and the observed dispersion in the high-frequency power-law component. Another possible difference between the two ACF classes is hinted at by the trends shown in Fig. 10, which could be interpreted as an indication that broad ACF bursts show less spectral evolution than the narrow ones. This view is indirectly supported by the comparison of the respective median PDS $\tilde{P}_{f}$ at different energies estimated in Sects. 4.2 and 5.2. The narrow class exhibits a single power-law PDS with index $\alpha_{\gamma}^{(\mathrm{n})} \approx 2$ at high energies and a steeper $\alpha_{\mathrm{X}}^{(\mathrm{n})} \approx 3$ low energies. In shot noise models where LCs are generated by random pulses, an index 2 commonly appears when the pulses have sharp rising phase, like the typical FRED-like pulses in many GRBs. But due to the hardto-soft evolution (Borgonovo \& Ryde 2001) the same pulses at $\mathrm{X}$-ray energies look smoother with slower rise phases giving steeper indices. On the other hand, the high-frequency powerlaw component in the broad class PDS shows approximately the same index in both energy bands (close to the 5/3 Kolmogorov index). Visual inspection in the $\gamma$ and X-ray bands of the bright broad ACF case GRB 990123 (see, e.g., Corsi et al. 2005), which shows well-separated pulses, indicates that the broadening of the ACF at low energies is not as much due to a corresponding smoothing of individual short pulses as to a decrease in their relative importance with respect to a long smooth LC component that seems to lie underneath them. If this case were representative of the whole class then that would explain in terms of LC components the observed $\tilde{P}_{f}$ behaviour at different energies.

One remarkable feature of the found ACF bimodality is the low relative dispersion of the broad class ( $\sim 6 \%$ in the $\gamma$ band) around the $\bar{w}_{\gamma}^{\prime(b)}=(7.42 \pm 0.14) \mathrm{s}$ timescale. If confirmed such a temporal feature would have very important implications, both for our physical understanding of GRBs and because of its potential use in cosmological studies. A crucial issue then is to properly account for redshift dependencies. The reported ACF widths were derived taking into account only the cosmic time dilation, since other effects are of a lesser order. The latter were quantified by means of the free parameter $a$ included in Eq. (3) width transformation. We found well-defined minima at $a_{\text {min }}^{(\mathrm{n})}=-0.35 \pm 0.2$ and $a_{\text {min }}^{(\mathrm{b})}=0.04 \pm 0.04$ for the narrow and broad ACF class respectively (in agreement with B04). Since F95 reported a considerable energy dependence for the average $\mathrm{ACF}\left(w(E) \propto E^{-0.43}\right)$ then the cosmic energy shift would be able to account within errors for the found $a_{\min }$ in the narrow class. However, for the broad class either we would need to assume that there are other dependencies at work that coincidentally cancel out almost exactly the energy shift effect or that simply the ACF energy dependence is weaker in this case. From the 
analysis of this alternative presented in Sect. 7 we conclude that in the context of our ACF study the power-law energy dependence is better characterised by a median index $\tilde{\xi}_{2 \mathrm{ch}}=0.21$, still consistent with the narrow class within errors and a considerably smaller effect to account for. We evaluated by Monte Carlo simulations that the estimated $w^{\prime}$ dispersion (for a broad class sample of only 7 bursts) is smaller but not significantly different to the one expected assuming the $\xi_{2 \mathrm{ch}}$ distribution found in Fig. 9. Moreover, we found indications that the broad ACF class may have lower typical $\xi$ indices than the general sample. This would be an expected consequence of the weaker spectral evolution already mentioned above when comparing the two classes.

Based on the analysis of WFC X-ray data, Vetere et al. (2006) reported that in a fraction of bursts the existence of two LC components is apparent. They selected 10 bursts by visual inspection and found for their slow/fast (low/high frequency variability) LC decomposition that the slow components were in general softer than the corresponding fast ones, suggesting different underlying emission processes. There are only 3 GRBs that they claim to have slow components among our sample with known $z$, but they all show broad ACFs in the source rest-frame (including the outlier GRB 010222), which suggests a connection to our own results that will need further analysis to be established. The discussion about the nature of the slow/fast components in Vetere et al. (2006) would apply also to the low/high frequency components found in broad ACF bursts. Within the framework of the internal shock model developed by Mészáros $\&$ Rees (2000), there would be two major radiating regions in the relativistic outflow and the PDS components would arise from the different expected variability of the emission, i.e., from a thermal photospheric component and from the non-thermal flux of optically thin dissipative regions above the photospheric radius. In this context, ACF class differences could be due to the conditions controlling the relative strength of those two emission components (Ryde et al. 2006).

Acknowledgements. We thank S. Larsson and C.-I. Björnsson for useful comments and careful reading of the manuscript. We are grateful to the anonymous referee for constructive criticism. This research has made use of BATSE and Konus data obtained from the High Energy Astrophysics Science Archive Research Center (HEASARC), provided by NASA's Goddard Space Flight Center.

\section{References}

Amati, L., Frontera, F., Vietri, M., et al. 2000, Science, 290, 953

Amati, L., Frontera, F., Tavani, M., et al. 2002, A\&A, 390, 81

Andersen, M. I., Hjorth, J., Pedersen, H., et al. 2000, A\&A, 364, L54

Antonelli, L. A., Piro, L., Vietri, M., et al. 2000, ApJ, 545, L39

Aptekar, R. L., Frederiks, D. D., Golenetskii, S. V., et al. 1995, Space Sci. Rev., 71,265
Belli, B. M. 1992, ApJ, 393, 266

Beloborodov, A. M., Stern, B. E., \& Svensson, R. 1998, ApJ, 508, L25 Beloborodov, A. M., Stern, B. E., \& Svensson, R. 2000, ApJ, 535, 158

Beuermann, K., Hessman, F. V., Reinsch, K., et al. 1999, A\&A, 352, L26

Bloom, J. S., Kulkarni, S. R., Djorgovski, S. G., et al. 1999, Nature, 401, 453

Bloom, J. S., Djorgovski, S. G., \& Kulkarni, S. R. 2001, ApJ, 554, 678

Bloom, J. S., Berger, E., Kulkarni, S. R., Djorgovski, S. G., \& Frail, D. A. 2003, AJ, 125,999

Borgonovo, L. 2004, A\&A, 418, 487 (B04)

Borgonovo, L., \& Ryde, F. 2001, ApJ, 548, 770

Borgonovo, L., \& Björnsson, C.-I. 2006, ApJ, 652, 1423

Borgonovo, L., Frontera, F., Guidorzi, C., Montanari, E., \& Soffitta, P. 2005, Il Nuovo Cimento, 28 C, 275

Corsi, A., Piro, L., Kuulkers, E., et al. 2005, A\&A, 438, 829

Costa, E., Frontera, F., Heise, J., et al. 1997, Nature, 387, 783

Djorgovski, S. G., Kulkarni, S. R., Bloom, J. S., et al. 1998, ApJ, 508, L17

Djorgovski, S. G., Frail, D. A., Kulkarni, S. R., et al. 2001, ApJ, 562, 654

Dodonov, S. N., Afanasiev, V. L., Sokolov, V. V., Moiseev, A. V., \& CastroTirado, A. J. 1999, GCN Report 475

Fenimore, E. E., int't Zand, J. J. M., Norris, J. P., Bonnell, J. T., \& Nemiroff, R. J. 1995, ApJ, 448, L101 (F95)

Fishman, G. J., et al. 1989, in Proc. of the GRO Science Workshop, ed. W. N. Johnson, 2

Friedman, A. S., \& Bloom, J. S. 2005, ApJ, 627, 1

Frontera, F., Costa, E., dal Fiume, D., et al. 1997, A\&AS, 122, 357

Garnavich, P. M., Stanek, K. Z., Wyrzykowski, L., et al. 2003, ApJ, 582, 924

Greiner, J., Peimbert, M., Estaban, C., et al. 2003, GCN Report 2020

Horváth, I. 1998, ApJ, 508, 757

Jager, R., Mels, W. A., Brinkman, A. C., et al. 1997, A\&AS, 125, 557

Jha, S., Pahre, M. A., Garnavich, P. M., et al. 2001, ApJ, 554, L155

Kolmogorov, A. 1941, Dokl. Akad. Nauk SSSR, 31, 538

Koshut, T. M., Kouveliotou, C., Paciesas, W. S., et al. 1995, ApJ, 452, 145

Kulkarni, S. R., Djorgoski, S. G., Ramaprakash, A. N., et al. 1998, Nature, 393, 35

Kulkarni, S. R., Djorgovski, S. G., Odewahn, S. C., et al. 1999, Nature, 398, 389 Lamb, D. Q., Castander, F. J., \& Reichart, D. E. 1999, A\&AS, 138, 479

Lazzati, D. 2002, MNRAS, 337, 1426

Liang, E., \& Zhang, B. 2006, ApJ, 638, L67

Link, B., Epstein, R. I., \& Priedhorsky, W. C. 1993, ApJ, 408, L81

Mészáros, P., \& Rees, M. J. 2000, ApJ, 530, 292

Metzger, M. R., Djorgovski, S. G., Kulkarni, S. R., et al. 1997, Nature, 387, 878

Miyamoto, S., Kitamoto, S., Iga, S., Negoro, H., \& Terada, K. 1992, ApJ, 391, L21

Muñoz, J. A., \& Tan, J. C. 2005, ApJ, 625, 256

Mukherjee, S., Feigelson, E. D., Jogesh Babu, G., et al. 1998, ApJ, 508, 314

Nardini, M., Ghisellini, G., Ghirlanda, G., et al. 2006, A\&A, 451, 821

Nakar, E., \& Piran, T. 2002, MNRAS, 331, 40

Norris, J. P., Nemiroff, R. J., Bonnell, J. T., et al. 1996, ApJ, 459, 393

Piro, L., Frail, D. A., Gorosabel, J., et al. 2002, ApJ, 577, 680

Press, W. H., Teukolsky, S. A., Vetterling, W. T., \& Flannery, B. P. 1992,

Numerical Recipes in Fortran, 2nd Ed. (Cambridge: Cambridge Univ. Press)

Price, P. A., Kulkarni, S. R., Berger, E., et al. 2002, ApJ, 571, L121

Reichart, D. E., Lamb, D. Q., Fenimore, E. E., et al. 2001, ApJ, 552, 57

Ryde, F., Björnsson, C.-I., Kaneko, Y., et al. 2006, ApJ, 652, 1400

Tinney, C., et al. 1998, IAU Circular 6896

Vetere, L., Massaro, E., Costa, E., Soffitta, P., \& Ventura, G. 2006, A\&A, 447, 499

Vreeswijk, P. M., Rol, E., Hjorth, J., et al. 1999, GCN Report 496

Vreeswijk, P. M., Fruchter, A., Kaper, L., et al. 2001, ApJ, 546, 672 